\title{
Reflets
}

Revue ontaroise d'intervention sociale et communautaire

\section{Alphabétisation et lutte contre la pauvreté chez les francophones d'Ottawa-Carleton}

\section{Colette B. Lacroix et Yasmine Nedjar}

Volume 3, numéro 1, printemps 1997

Enfance et familles en contexte d'appauvrissement

URI : https://id.erudit.org/iderudit/026159ar

DOI : https://doi.org/10.7202/026159ar

Aller au sommaire du numéro

Éditeur(s)

Reflets : Revue ontaroise d'intervention sociale et communautaire

ISSN

1203-4576 (imprimé)

1712-8498 (numérique)

Découvrir la revue

Citer cet article

Lacroix, C. B. \& Nedjar, Y. (1997). Alphabétisation et lutte contre la pauvreté chez les francophones d'Ottawa-Carleton. Reflets, 3(1), 153-157.

https://doi.org/10.7202/026159ar

Tous droits réservés (C) Reflets : Revue ontaroise d'intervention sociale et communautaire, 1997
Ce document est protégé par la loi sur le droit d'auteur. L'utilisation des services d'Érudit (y compris la reproduction) est assujettie à sa politique d'utilisation que vous pouvez consulter en ligne.

https://apropos.erudit.org/fr/usagers/politique-dutilisation/ 


\section{Alphabétisation et lutte contre la pauvreté chez les francophones d'Ottawa-Carleton}

\section{Colette B. Lacroix et Yasmine Nedjar}

La Magie des lettres, Ottawa

La Magie des Lettres est heureuse de faire une modeste contribution à la réflexion sur un sujet aussi grave que la pauvreté chez les enfants. Nous sommes confrontés quotidiennement à cette réalité aussi bien à travers le vécu de nos apprenants que dans les chiffres traitant de la problématique du décrochage scolaire. Nous espérons que les réflexions et les interventions présentées ici constitueront la base d'une nouvelle approche en vue d'une prise en charge plus responsable de ce problème par toutes les parties concernées.

L'augmentation du taux de pauvreté chez les enfants est causée en majeure partie par le fait que les lois du marché ne prévoient presque aucune protection contre la pauvreté. De plus, le désengagement des pouvoirs publics, notamment à travers les coupures opérées dans les services et ressources éducatives, favorise la montée de ce phénomène.

Si l'on se réfere aux années 1970, la plupart des jeunes familles pouvaient vivre du salaire d'une personne travaillant 40 heures par semaine. Aujourd'hui, les deux parents doivent travailler pour subvenir aux besoins familiaux et on note une augmentation de $37 \%$ du nombre de familles pauvres parmi les parents qui travaillent à temps plein. Ceci ne tient même pas compte du quart de million de personnes qui n'ont pas d'autre choix que de travailler à temps partiel. Plusieurs de ces emplois sont mal 
rémunérés, précaires et sans avantages sociaux. (Campagne 2000, 1995).

Tous les indices montrent que, dans la plupart des cas, la population des enfants pauvres s'accroît continuellement. Les enfants issus de milieux défavorisés débutent l'école avec un retard académique par rapport à leurs pairs mieux nantis. Dès le départ, ces «inégalités» ont des répercussions négatives sur leur estime de soi et diminuent leurs chances de réussite scolaire. Ce sont ces circonstances qui, très souvent, les amèneront à vivre des situations de décrochage, de chômage ou de sous-emploi.

C'est précisément pour briser ce cycle négatif associé au décrochage scolaire que la Magie des lettres a lancé le «Club de devoirs», destiné d'une part à aider les enfants à surmonter les difficultés d'apprentissage scolaire et, d'autre part, à les motiver socialement en encourageant leur estime de soi. Le «Club de devoirs» répond à des attentes nombreuses et élevées puisqu'il vise un des groupes les plus vulnérables de la population, c'est-àdire les enfants de 8 à 12 ans.

Ce programme se donne pour mission de réconcilier les enfants qui ont de la difficulté avec les études, en leur permettant de réviser le français et de faire leurs devoirs sur les ordinateurs de la salle «Richelieu» à la Magie des lettres. Il ne fait pas de doute que l'approche conviviale assignée à ce programme contribuera à rebâtir la confiance de ces enfants quant à leurs capacités de réussite scolaire d'autant plus que collation et jeux éducatifs font partie du menu du «Club de devoirs» qui a démarré à l'automne 1996.

Outre sa contribution sur le plan scolaire, ce «Club» tente de susciter l'implication de plusieurs des parties concernées par les problèmes de l'éducation, notamment, la communauté, les écoles et les parents. Son but est d'établir un partenariat dans lequel les participants s'engagent à réfléchir ensemble sur les moyens à mettre en oeuvre pour venir en aide aux enfants en difficulté et réduire les effets néfastes de la pauvreté des enfants.

Les parents sont les personnes ayant le plus d'influence sur les enfants. Ce sont eux qui les encouragent à développer les aptitudes, les compétences et les qualités nécessaires au succès aussi bien à 
l'école que dans la vie. Ce processus commence très tôt, avec l'apprentissage du langage et des aptitudes sociales. C'est pourquoi l'éducation est toujours une variable primordiale lorsqu'il s'agit d'enrayer le cycle de la pauvreté.

Les recherches démontrent que les enfants qui ont bénéficié de programmes préscolaires bien structurés et bien dirigés sont nettement mieux préparés aux plans académique, émotionnel, social et physique. De là l'intérêt de généraliser ces programmes et de permettre aux enfants de familles défavorisées de se familiariser très tôt avec la lecture, de s'éduquer au partage et aux règles de la vie en société.

Une étude réalisée au Michigan, dans laquelle des enfants d'âge préscolaire ont été suivis jusqu'à l'âge adulte, a révélé que chaque dollar investi dans un bon programme de la petite enfance représente une épargne de sept dollars en mesures de «redressement» éventuelles dans les services éducatifs, les dépenses judiciaires liées à la criminalité et les coûts d'aide sociale. Les conclusions de l'étude révèlent également qu'un citoyen instruit est essentiel à une économie moderne et compétitive.

Par conséquent, les coupures faites dans les ressources et les services offerts aux familles à faible revenu ont pour effet d'augmenter le pourcentage du nombre de familles «emprisonnées» dans le cycle de la pauvreté, les empêchant de réintégrer la classe moyenne et d'y demeurer. En 1991, selon Statistique Canada, $15 \%$ des francophones d'Ottawa-Carleton vivaient dans la pauvreté. La majorité de ces francophones habitaient les secteurs de Vanier et d'Ottawa. Cette situation ne peut que s'aggraver compte tenu du contexte économique actuel. D'où la nécessité pour la communauté de se mobiliser pour réfléchir aux moyens de lutter contre ce fléau.

À la Magie des lettres, nous avons décidé d'élargir la couverture des services par la mise sur pied du programme «alpha familiale». Ce nouveau programme permet d'intervenir auprès des jeunes enfants des familles défavorisées. Comme son nom l'indique, il vise à la fois l'épanouissement des enfants d'âge préscolaire et la valorisation du rôle des parents. La démarche préconisée consiste 
à briser l'isolement des parents analphabètes en les aidant à acquérir des techniques d'apprentissage et en les outillant de manière à ce qu'ils puissent développer des aptitudes chez leurs enfants. Ces derniers disposeront ainsi des ressources nécessaires pour poursuivre une meilleure éducation.

Convaincue de l'importance d'agir dès la petite enfance pour prévenir les situations de décrochage et les autres expériences négatives liées à l'analphabétisme, la Magie des lettres sensibilise la communauté à l'urgence de mettre en place des mesures de prévention pour lutter contre l'analphabétisme des adultes et contre la pauvreté des enfants.

Certes, la mise en oeuvre du programme nécessite des moyens humains et matériels que la Magie n'a pas encore réunis. D'ores et déjà, toute une équipe s'active à la préparation de ce projet. Qu'il s'agisse de la conception de trousses d'information ou de la mobilisation d'un formidable potentiel de bénévoles en passant par la collecte de matériel éducatif, tout est presque prêt pour que le programme démarre. Comme nous l'avons dit précédemment, les études démontrent qu'une intervention à la source du problème peut faire une différence. La Magie partage totalement ce point de vue.

En terminant, nous suggérons une lecture des points importants contenus dans le document Investir dans les générations à venir (Campagne 2000 et la réforme de la sécurité sociale) qui propose les solutions suivantes :

- Une stratégie «des cycles de vie» qui procure le soutien nécessaire aux familles lors des périodes de transition difficile (donc aussi bien aux futurs parents qu'aux parents et aux enfants). Cette approche préventive permet de remédier aux situations qui rendent les gens vulnérables. Sur le plan financier, cette stratégie doit donc surtout viser les familles à revenus modestes ou moyens, plus vulnérables aux changements qui entraînent la pauvreté.

- Un ensemble de politiques et de programmes intégrés et cohérents dans trois domaines qui constituent les fondements de la stratégie des cycles de vie, soit:

- Une stratégie nationale de développement des perspectives 
d'emploi, y compris un revenu minimum suffisant, un remaniement des tâches et un partage du travail, une compensation financière pour les personnes s'occupant d'enfants et de personnes âgées, des bénéfices sociaux pour les employés à temps partiel, des congés spéciaux de formation et des congés parentaux.

- Un système de sécurité sociale adéquat, y compris un système progressif de prestations pour enfants, un système garanti de pensions alimentaires pour les enfants en cas de divorce ou de séparation, un système de garde complet, un programme national de bourses d'études postsecondaires, un système de garantie de revenu pour les travailleurs en période de transition.

- Un système d'aide communautaire qui répond aux besoins des gens, y compris un service d'éducation en matière de santé, de soins pré et postnataux, de garderie et de soutien aux familles, de partenariat avec les écoles et des possibilités de «rattrapage» pour que les jeunes finissent leurs études secondaires.

La pauvreté des enfants est un phénomène résultant d'une dégradation du tissu social. Sa résorption dépend de la mise en place d'une stratégie globale visant les familles à revenus modestes ou moyens, et identifiées comme les plus vulnérables face aux bouleversements économiques à l'origine de la pauvreté. Autrement dit, c'est en s'attaquant au problème de l'emploi et à la réforme du système de sécurité sociale en vue de mieux protéger les familles fragiles au plan économique que la pauvreté des enfants diminuera dans nos communautés. 